This is an electronic reprint of the original article. This reprint may differ from the original in pagination and typographic detail.

Author(s): Laitinen, Mikko; Sajavaara, Timo

Title: $\quad$ Trajectory bending and energy spreading of charged ions in time-of-flight telescopes used for ion beam analysis

Year: $\quad 2014$

Version:

Please cite the original version:

Laitinen, M., \& Sajavaara, T. (2014). Trajectory bending and energy spreading of charged ions in time-of-flight telescopes used for ion beam analysis. Nuclear Instruments \& Methods in Physics Research. Section B: Beam Interactions with Materials and Atoms, 325(April), 101-106.

https://doi.org/10.1016/j.nimb.2014.01.015

All material supplied via JYX is protected by copyright and other intellectual property rights, and duplication or sale of all or part of any of the repository collections is not permitted, except that material may be duplicated by you for your research use or educational purposes in electronic or print form. You must obtain permission for any other use. Electronic or print copies may not be offered, whether for sale or otherwise to anyone who is not an authorised user. 


\title{
Trajectory bending and energy spreading of charged ions in time-of-flight telescopes used for ion beam analysis
}

\author{
Mikko Laitinen, Timo Sajavaara \\ Dept. of Physics, P.O.Box 35, FI-40014 University of Jyväskylä, Finland \\ Corresponding author: Mikko Laitinen \\ Telephone : +358400994836 \\ Fax: +358 14617411 \\ E-mail: mikko.i.laitinen@jyu.fi
}

\begin{abstract}
:
Carbon foil time pick-up detectors are widely used in pairs in ion beam applications as time-of-flight detectors. These detectors are suitable for a wide energy range and for all ions but at the lowest energies the tandem effect limits the achievable time of flight and therefore the energy resolution. Tandem effect occurs when an ion passes the first carbon foil of the timing detector and its charge state is changed. As the carbon foil of the first timing detector has often a non-zero voltage the ion can accelerate or decelerate before and after the timing gate. The combination of different charge state properties before and after the carbon foil now induces spread to the measured times of flight. We have simulated different time pick-up detector orientations, voltages, ions and ion energies to examine the tandem effect in detail and found out that the individual timing detector orientation and the average ion charge state have a very small influence to the magnitude of the tandem effect. On the other hand, the width of the charge state distribution for particular ion and energy in the first carbon foil, and the carbon foil voltage contributes linearly to the magnitude of the tandem effect. In the simulations low energy light ion trajectories were observed to bend in the electric fields of the first timing gate, and the magnitude of this bending was studied. It was found out that $50-150 \mathrm{keV}$ proton trajectories can even bend outside the second timing gate.
\end{abstract}

Keywords: tandem effect, carbon foil, Time-of-Flight, ToF-E, ToF-ERDA 


\section{Introduction}

Time-of-flight (ToF) telescope comprising two carbon foil time pick-up detectors (for short: timing gates) is one of the most versatile and useful detectors in the field of ion beam analysis. It can be used for all ions and usable energy regime extends down to few tens of keVs [1]. The energy resolution of the ToF-system for monoenergetic ions depends from the individual timing accuracy of the detectors, the distance of the flight path, the energy straggling and the thickness variation of the first carbon foil and also from the tandem effect [2], to list a few. The performance of the MCP, anode $[3,4]$ and the isochronous electron transportation $[5,6]$ in the timing gates contribute also to the energy resolution. Another additional factor is the timing gate orientation [7] in which, for example, the forward emitted electrons have increased probability for higher energies in wider emission angles [8] which will lead to non-isochronous electron transportation from the foil to the MCP. The better known limitations of the ToF-detectors are the detection efficiency for hydrogen and low energy heavy ion scattering in the carbon foil [9] of the first timing gate (T1). One additional limitation which comes in question with the tandem effect is the use of high or low voltages: when using the MCP for electron amplification, either the carbon foil or anode needs to be at elevated voltage. If the carbon foil is in high voltage and enhances the tandem effect, then the anode can be grounded. The grounded foil -situation requires an anode in high voltage while it also increases the background events because of the free electrons are then more easily accelerated towards the MCP.

When high depth resolution for thin film sample analyzes is aimed for elastic recoil detection telescopes equipped with ToF it is beneficiary to use lower energies [10]. In lower energies, however, one has to also consider the tandem effect [2] and the bending of the ion trajectories in the electric fields of the first timing gate. The tandem effect can generally be described as a time-of-flight (and energy) spread due to the charge state exchange of the passing ion in the carbon foil of the first timing gate. This phenomenon is often listed as one of the most important factors limiting the resolution of ToF detectors for ion beam analysis using the lowest beam energies [2,10--12]. The additional energy spread of the passing ion caused by the tandem effect has often been written in a form: $\sigma_{\text {tandem }}=\overline{\Delta q} \cdot V_{\mathrm{T} 1 \text { foil }}$, where $\overline{\Delta q}$ is the average change of the charge of the ions upon their passage through the $\mathrm{T} 1$ foil having voltage of $V_{\mathrm{T} 1 \text { foil }}$. However, further quantification of the $\overline{\Delta q}$ has generally not been explained.

For low energy charged ions also notable bending of ion trajectories takes place in the time pick-up detectors due to electric fields and can even lead to a situation that no ions reach the second timing gate at all if both gates have strictly limited solid angles.

We have simulated with SimION software [13] different timing gate configurations and measured experimentally results with the time-of-flight elastic recoil detection (ToF-ERD) spectrometer in Jyväskylä to test and to gain better knowledge of the ion trajectory bending and the tandem effect. The aim was to find the optimal timing gate design, if not the one used at the moment in the view of bending of ion trajectories and the tandem effect. 


\section{Simulation and experimental setups}

94 Simulation software used was SimION [13] which is a 3D capable electron and ion transport simulation program. The time-of-flight telescope dimensions were replicated to the simulation program from the real ToF-ERD system existing in our laboratory. The wire grids in the timing gates were modeled as transparent potential barriers. Similar simplification was applied also to the carbon foils.

Most important assumption made in this study is that the charge exchange equilibrium is

101

102 always reached for energetic $(>50 \mathrm{keV})$ ions in both T1 carbon foil $\left(\sim 3 \mu \mathrm{g} / \mathrm{cm}^{2}\right)$ and in

103

104

105

106 the sample from which the ions, scattered or recoiled, emerge towards the ToF-E telescope. There are numerous publications detailing with the charge exchange processes for different targets, incident ions and energies but for the essentials, a general illustration of the field is summed well in [14] and a more specific case for lower energies is presented in [15]. In these references it is shown that about $1 \mu \mathrm{g} / \mathrm{cm}^{2}$ of material is already enough for $\mathrm{MeV}$ ions to reach the charge state equilibrium. If this statement is expected to be valid then the charge states of the ions incoming to the first time pick-up detector and leaving from the $\mathrm{T} 1$ foil are independent of each other but follow the same energy dependent charge state distribution. This means that for the He ions, for example, there are total $3 \times 3$ different charge state combinations for the ions that have emerged from the sample and passed the $\mathrm{T} 1$ foil and thus 9 different ToFs can exist after the T1 foil for the He ions.

116 Figure 1. Different timing gate orientations. In all simulations the distance from foil-to117 foil was kept the same. The both timing gate carbon foils in a) face tow ards the $E$ detector, 118 in b) face off from each other, in c) face towards the beam and in d) face towards each 119 other.

120 During the simulations, the timing gate orientations (see Fig. 1) and voltages were 121 changed to examine how the transmitted ions behave in different configurations. In 122 simulations, when non-zero voltage was applied to the $\mathrm{T} 1$ carbon foil, it was assumed 123 that the incoming ions had scattered/recoiled from a sample in ground potential and 124 reached the charge state equilibrium characteristic for that particular energy. The charge 125 state distributions for different energies were taken from the tables of comprehensive 126 database [16] where different ion-target combinations are summed up from numerous 127 publications from the past decades. For this study, only carbon foil as a target (=T1 foil) 128 material was considered. 
130 Experimental setup consists of a sample located at the distance of $32 \mathrm{~cm}$ from the first 131 timing gate, particle suppressors, two carbon foil time pick-up detectors similar to the 132 design of Busch et al. [6] and with the time-of-flight distance of $62 \mathrm{~cm}$. After the second 133 timing gate there is a silicon energy detector allowing coincident ToF-E measurements 134 and thereafter mass identification of the individual particles. The foils of the both time 135 pick-up detectors in the experimental setup are facing towards the energy detector (like in 136 Fig. 1 a). Typical voltages in the experimental setup are: $-500 \mathrm{~V}$ for the $\mathrm{T} 1 \mathrm{foil}$ and 137 mirror, $+1000 \mathrm{~V}$ for the toblerone (which is the triangular part creating a field-free region) 138 and $\mathrm{MCP}_{\text {in }}$ and $+2750 \mathrm{~V}$ for the anode; $-2800 \mathrm{~V}$ for the T2 foil and mirror, $-1800 \mathrm{~V}$ for 139 toblerone and $\mathrm{MCP}_{\text {in }}$ and $0 \mathrm{~V}$ for the anode, similar as shown in the Fig. 2 b).

\section{Results}

When the full ToF simulations were run with different ions, two significant results could easily be identified. First was the clear bending of light, low energy ion trajectories in the electric field of the first timing gate mirror grid/harp. The second was the observation that even with zero voltage at the T1 carbon foil, or even at both T1 and T2 foils, the charge

147 state dependent tandem effect did not vanish completely. From the simulations it also 148 became evident that using the existing timing gate configuration of the ToF-E telescope 149 no concrete experimental evidence could be obtained of the tandem effect. This was due 150 to the limited maximum achievable voltage of the T1 foil $(\sim 1250 \mathrm{~V})$ in our operating 151 ToF-ERDA configuration. Thus results (all results given are FWHM) presented here are 152 all based on simulations.

\subsection{Bending of the light ions due to timing gate voltages}

The bending of ion trajectories in the T1 timing gate was most prominent for the hydrogen ions at the lowest simulated energies (50-150 keV). As can be seen from the

158 Fig. 2, the ion trajectories bend more when the T1 foil and mirror grid voltages are set to $1590 \mathrm{~V}$ and $-500 \mathrm{~V}$, respectively (see Fig. $2 \mathrm{~b}, \mathrm{c}$ and d) compared to the situation when the 160 foil and mirror are at $-2800 \mathrm{~V}$ as in Fig. 2 a). This is because of the potential difference 161 over at mirror grids is smaller in the latter case $(1000 \mathrm{~V})$ compared to the first case (Fig. $1622 \mathrm{~b}$ )), where the toblerone voltage is $+1000 \mathrm{~V}$ (1500 V difference over mirror grids). 


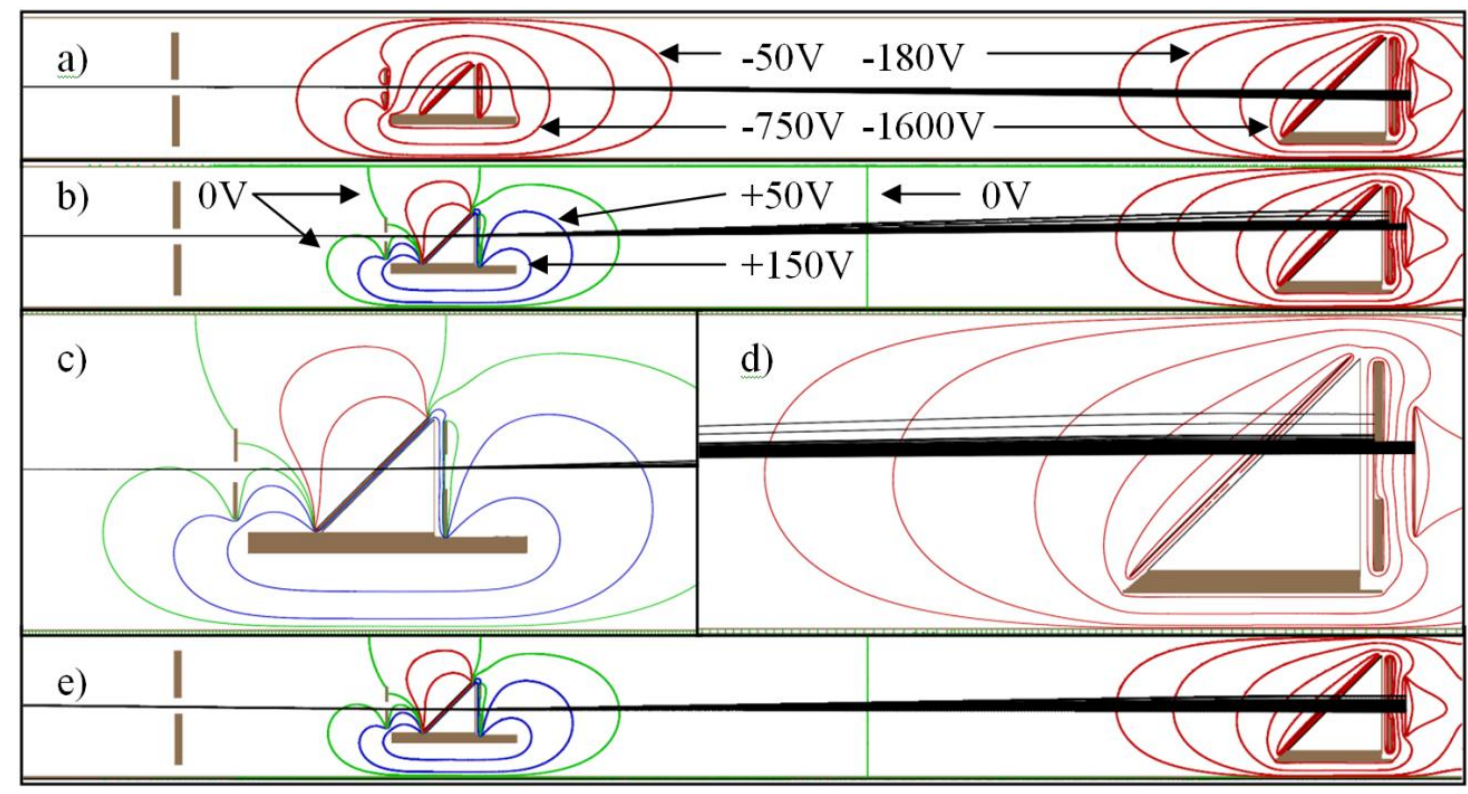

165 Figure 2. Bending of hydrogen ion trajectories in timing gates. Low energy hydrogen ion 166 paths (initial energy $100 \pm 50$ ) keV ) were simulated with SimIO N through the T oF-ERD 167 spectrometer with E-detector being further right after the T2 timing gate. Red color: negative potential, green col or: $0 \mathrm{~V}$ potential and blue color: positive potential. In a) both $\mathrm{T} 1$ and $\mathrm{T} 2$ have foil and mirror voltage at $-2800 \mathrm{~V}$ and virtually field free toblerone part at $-1800 \mathrm{~V}$. In the rest of the figures the $T 1$ foil is at the ground potential, the mirror at $500 \mathrm{~V}$ (to repel free electrons as in experimental setup) and the toblerone part for the T1 at $+1000 \mathrm{~V}$. O ne can see that in b) and in the close-ups of c) and d) the hydrogen paths for lowest energies will not end up to the E-detector when the foil opening at T2 is $18 \mathrm{~mm}$ 174 diameter. In e) 1\% downward declination is used for incoming hydrogen ions with 175 respect to the straight line of sight.

As seen from Fig. 2 d), the bending of the light ion trajectories can induce a reduction of the detection efficiency, which is already hindered due to the small stopping force and therefore small number of secondary electrons emitted by the lightest ions from carbon foils. However, this situation only occurs if the apertures before the first timing gate are limiting the solid angle too much. Example is given in Fig. 2 e) where incident ions that would not have hit the $\mathrm{T} 2$ detector originally are now bent in $\mathrm{T} 1$ towards the T2 detector. Due to this effect, if the first aperture(s) do not limit the solid angle too much, the number of missed ions, due to bending at $\mathrm{T} 1$, is compensated by the same number of ions that would have originally missed the T2 but are now bent towards it. This is the reason why the solid angle of the timing telescope should not be limited by the aperture(s) before the

190 A charge state exchange in T1 foil will induce an energy spread to incident 191 monoenergetic ions. The width of this spread caused by the tandem effect depend on 
three main parameters: a) T1 timing gate foil voltage, b) the ion proton number $(\mathrm{Z}), \mathrm{c})$ 193 velocity (energy) of specific ion. The charge state equilibrium and distribution which are 194 reached at the T1 foil, are actually bound to the combination of b) and c). As shown later, 195 timing gate orientation and other voltages than the one at T1 carbon foil have only a

198 The equilibrium charge state fraction $F_{q}\left(\sum_{q} F_{q}=1\right)$ defines the charge state probability of individual charge state before and after the foil. The width $d$ of this charge state distribution over all charge states can be defined through the average charge state $q_{a v}\left(=\sum_{q} q * F_{q}\right)$ being $d^{2}=\sum_{q}\left(q-q_{a v}\right)^{2} * F_{q}$. The values $q_{a v}$ and $d$ are the most usually reported values for the charge states distribution measurements for different ions

\begin{tabular}{|c|c|c|c|c|c|}
\hline Heenergy & Chan & tate d & ibution & 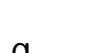 & $d$ \\
\hline [keV] & $2+$ & $1+$ & 0 & & \\
\hline 150 & 0.05 & 0.60 & 0.35 & 0.70 & 0.56 \\
\hline 250 & 0.12 & 0.64 & 0.24 & 0.88 & 0.59 \\
\hline 400 & 0.30 & 0.60 & 0.10 & 1.20 & 0.60 \\
\hline 600 & 0.51 & 0.46 & 0.03 & 1.48 & 0.56 \\
\hline 800 & 0.6 & 0.32 & 0.01 & 1.66 & 0.49 \\
\hline
\end{tabular}

205 Table I. Charge state fraction distribution of $\mathrm{He}$ after the carbon foil. $\mathrm{V}$ alues estimated 206 from a graph presented in [17]. qav is the average charge state and $d$ is the width (sigma) 207 of the distribution (values calculated from the charge state distribution).

208 By knowing the fractions for different individual charge states $F_{q}$, the 9 different ToFs of 209 the He form close to Gaussian shape when time-of-flights are simulated from the T1 foil 210 to the T2 detector foil. The original charge states emerging from the sample can change 211 in $\mathrm{T} 1$ foil and the resulting probability distribution of 9 different ToFs for the $250 \mathrm{keV} \mathrm{He}$ 212 ions are shown in Fig. 3. Here, it can be seen from the Table I, that the central peak of the 213 Gaussian shaped spread mainly forms from the 1+ to 1+ charge state exchange in the T1 214 foil. T simulation results shown in Fig. 3 for two different T1 timing gate voltages, and 215 T2 voltages kept the same, demonstrate that the ToF spread reduces for smaller T1 foil 216 voltages. 


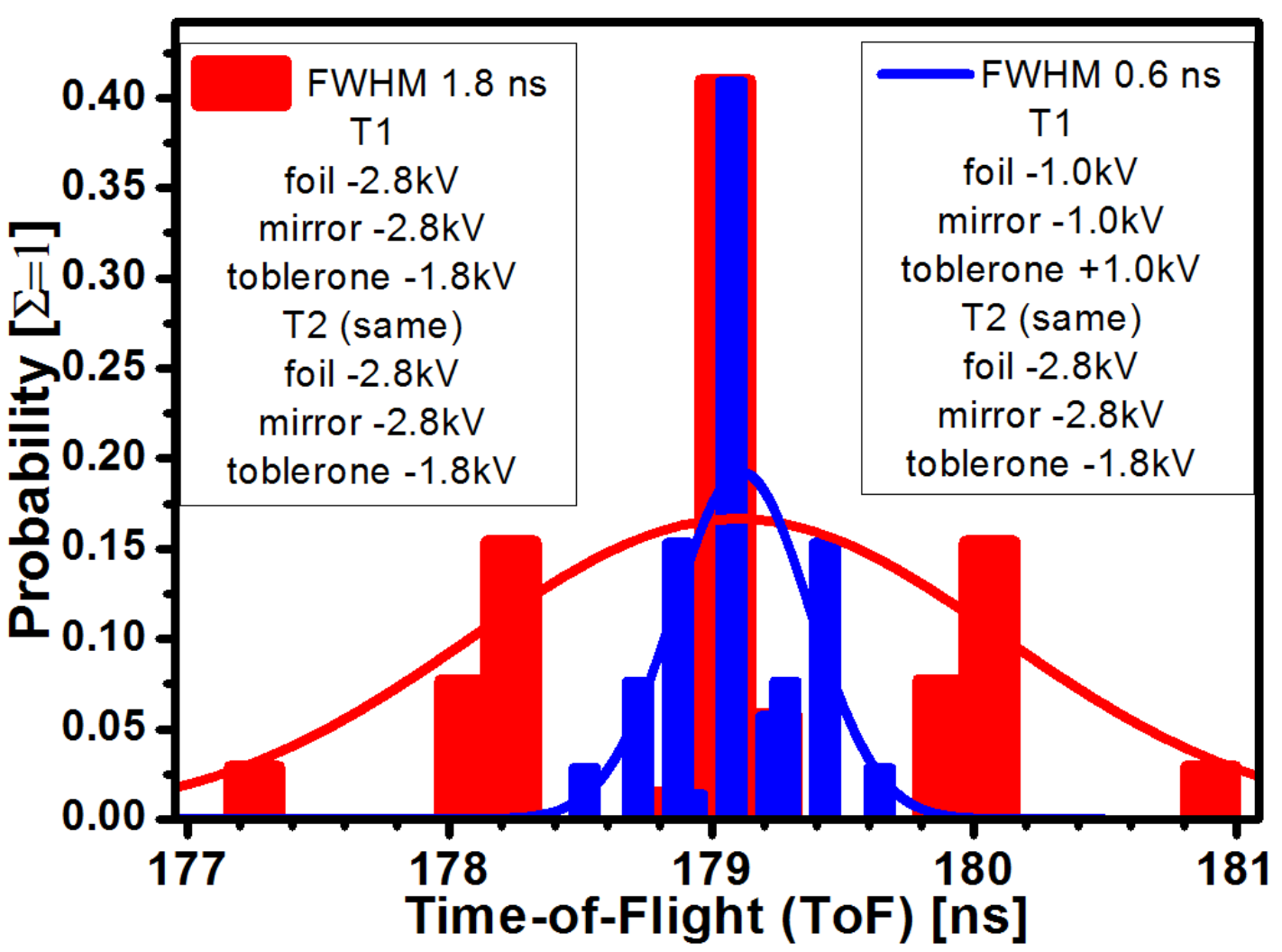

220 Figure 3. Simulated tandem effect induced time-of-flights for monoenergetic $250 \mathrm{keV} \mathrm{He}$ 221 ions with three different charge states. Correct $250 \mathrm{keV} \mathrm{H} \mathrm{e}$ time-of-flight is $179.23 \mathrm{~ns}$. 222 Both timing gates faced towards the energy detector. Fractions for different charge states 223 are taken from Table I. Fitted curves are pure Gaussian, FW H M s are calculated through 224 weighted standard deviation $\sigma$ obtained directly from the data.

In a similar manner as in Fig. 3, different timing gate orientations (see from Fig. 1) were simulated with varying foil and mirror voltages, in both timing gates same voltages were used. In practice, as can be seen from the Fig. 4, no differences in the magnitude (ToF spreading) of the tandem effect were observed between the four different timing gate orientations. However, small differences were observed when the peak position of the Gaussian fit of the different time-of-flights were compared. From this comparison, the timing gate orientation where foils faced towards each other suffered from the smallest position shift compared to the correct ToF of the selected energy. This shift of the peak position is however in practice always small compared to the magnitude of the simulated tandem effect. The spread induced by the tandem effect on the other hand can be close to $5 \%$ of ToF for low energies, if the foil voltages are pushed up to $7 \mathrm{kV}$ (see Fig. 4). 


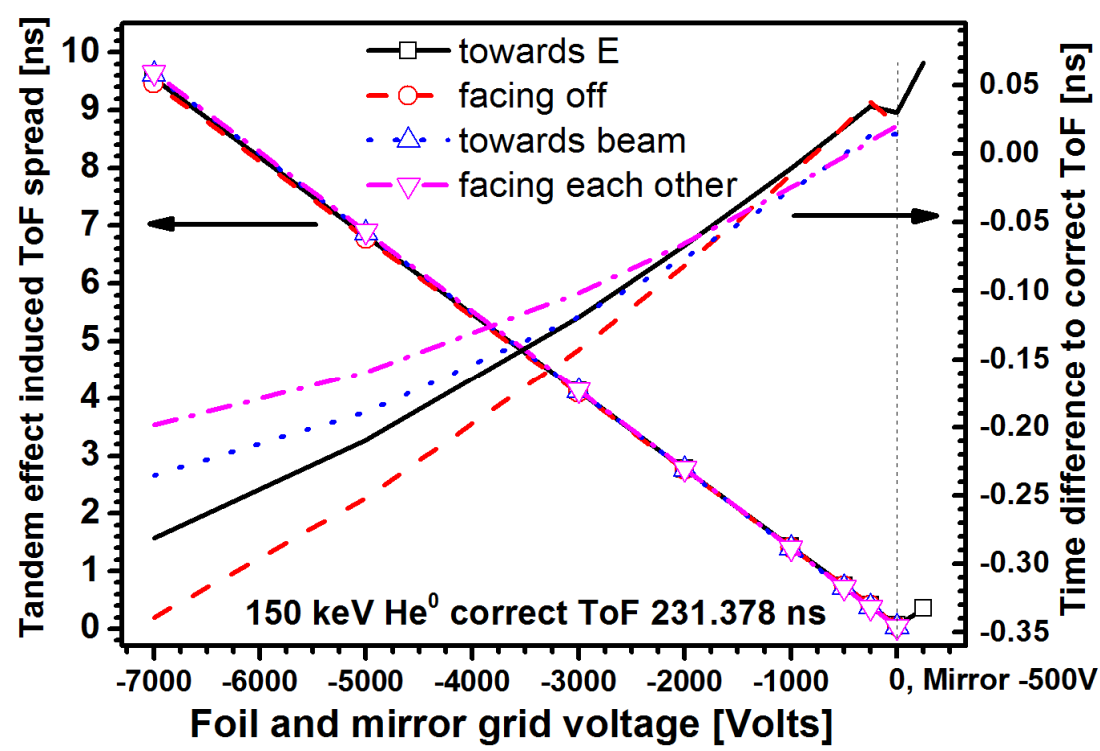

238 Figure 4. Impact of the timing gate orientation to the tandem effect induced ToF spread 239 (left $y$-axis) and peak position shift (right $y$-axis). Both timing gates had the same 240 voltages in all configurations. Simulated ion beam was $150 \mathrm{keV} \mathrm{He}$ and tobleronepart 241 voltage was $+1000 \mathrm{~V}$ for both timing gates. Timing gate orientation schematics can be 242 seen from Fig. 1.
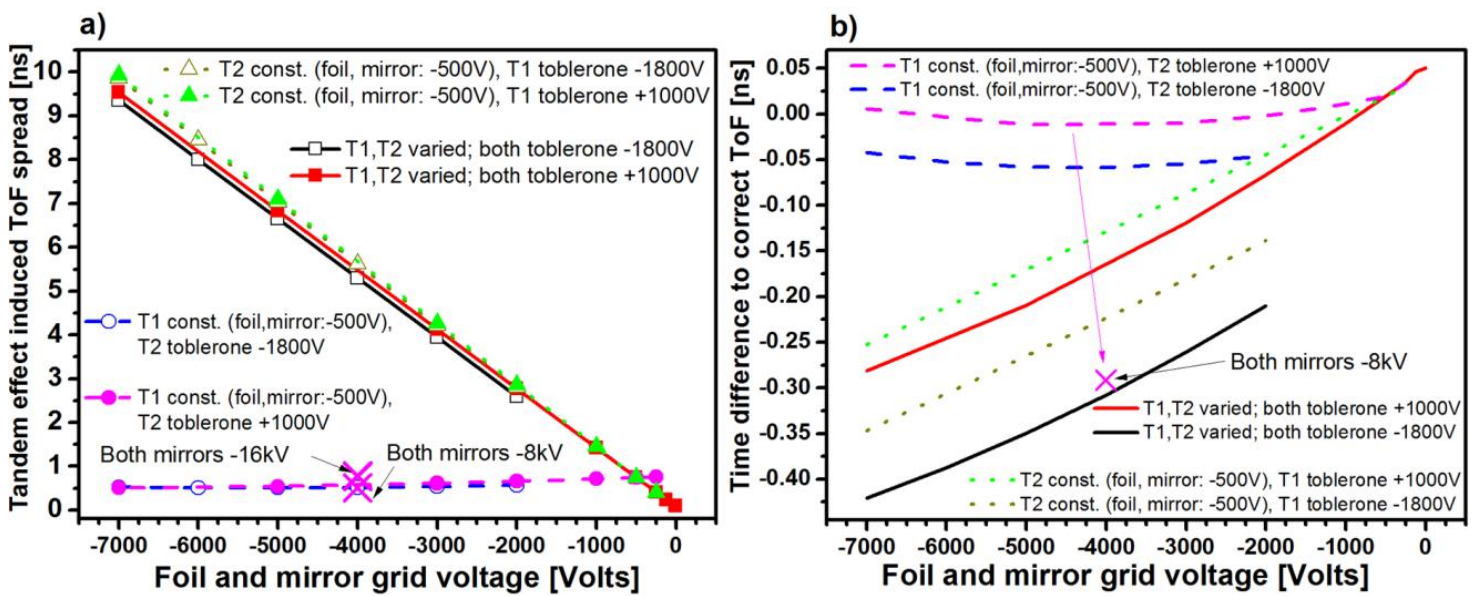

244 Figure 5. Impact of timing gate voltages to the tandem effect induced ToF spread (a) and 245 peak position shift (b). In a) the simulated spread is given as FWH M. Simulated $150 \mathrm{keV}$ 246 He ions were used and correct ToF for $\mathrm{H} \mathrm{e}^{0}$ is $231.378 \mathrm{~ns}$. The foil in both timing gates 247 was facing towards the energy detector. Constant voltages in a) and b) refer to $+1000 \mathrm{~V}$ 248 in toblerone and $-500 \mathrm{~V}$ in mirror and foil. Crosses where both mirrors are at high 249 voltage, $-8 \mathrm{kV}$ or $-16 \mathrm{kV}$, refer to the situation where $\mathrm{T} 1$ and both mirror voltages are 250 constant and $\mathrm{T} 2$ foil voltage is $-4000 \mathrm{~V}$ and toblerone $+1000 \mathrm{~V}$. 
251 In Fig. 5, the voltage configurations of T1 and T2 were varied to see how much does the 252 T2 or mirror grid voltages influence the tandem effect. In situations where other timing 253 gate is said to be constant, say T1 is constant, only T2 foil and mirror voltage were 254 changed. In Fig. 5 a) the cases where T1 foil voltage is kept constant, the tandem effect is small and causing less than $1 \mathrm{~ns}$ ToF spread even for the wide T2 foil voltage values. It can also be noted that the two points marked in the same figure whit text "both mirrors" at $-8 \mathrm{kV}$ or $-16 \mathrm{kV}$, do not influence the tandem effect in this timing gate configuration. Mirror voltages can be seen to have an effect only at Fig. $5 \mathrm{~b}$ ) where time difference to the correct $\mathrm{ToF}$ is compared. Also, the toblerone part voltage has only a minor influence to the tandem effect in both Fig. 5 a and b). In the Fig. 5 a) it is shown that when T1 foil has the lowest voltages the tandem effect is small. At the bottom part of the same Fig. 5 a), however, it can be seen that the $\mathrm{T} 2$ voltages do have an influence to the velocity (energy) profile of the passing ion when it flies between the two timing gates: for small, constant T1 voltage, the higher the T2 voltage is the smaller the tandem effect is. It is also clear that mirror grid voltages have no effect to the tandem effect but only the central ToF peak position will change with increasing mirror voltages. This is due to accelerating/decelerating effect of the T2 mirror grid (or also T1 depending on the gate orientations) while the ion is between the timing gate foils.

To analyze the ToF (and energy) spread induced by the of the tandem effect deeper, ions with different masses and energies were simulated with different timing gate orientations and voltages. In Fig. 6 a) it is shown that for different energies and different timing gate parameters the differences in the ToF spread values between the given situations stays roughly constant over a wide energy range for the He ions as well as for $\mathrm{C}$ ions. When simulated energy resolution (spread) for $\mathrm{He}$ and especially for heavier elements are compared (see Figs. 6 b, c, d), certain constant pattern on the tandem effect behavior can be seen. Fig. 6 b) shows that for the same ion $(\mathrm{He})$ over a wide energy range the tandem effect depends almost solely from the T1 foil voltage. In $6 \mathrm{c}$ ), the same linear dependence can be observed, but now the $\mathrm{Z}$ of the ion sets the magnitude of the tandem effect when the T1 foil voltage is kept constant. Finally when analyzing Fig. 6 d) the spread induced by the tandem effect is not only a function of the T1 foil voltage and mass of the ion but it follows almost hand-in-hand the width of the charge state distribution parameter $d$ over the very wide energy range, as can be expected. 

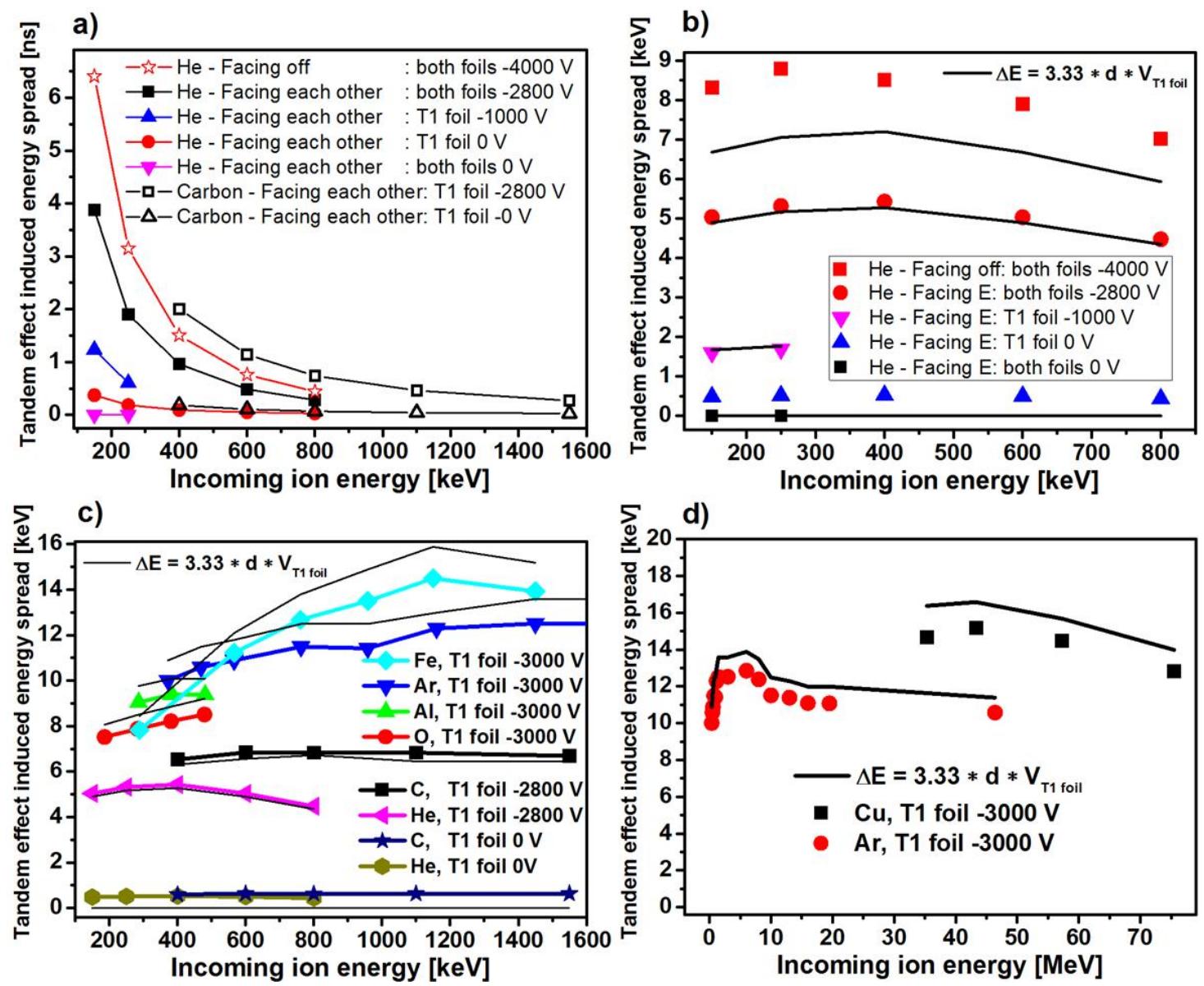

Figure 6. Simulated resolution degradation in nanoseconds (a) and keV s (b, c and d) due

286 to the tandem effect for different ion masses and beam energies. T1 mirror voltage is kept same as T1 foil and T 1 toblerone being $+1000 \mathrm{~V}$ while T 2 voltages are the same as in Fig.

2892 and 3. The $y$-axis are in FWH M. A Iso an estimation for the tandem effect is shown in 290 b), c) and d) as a solid line. The charge state fractions for different ions used in the 291 simulations are taken from the following references: He [17], C [18], O [19], AI [19], $292 \mathrm{Ar}(<1.5 \mathrm{M} \mathrm{eV}$ [18],> 1. $5 \mathrm{M} \mathrm{eV}$ [20]), Fe [18], Cu [21].

Values from the formula (1) estimating the magnitude of the tandem effect, are plotted to the Fig. 6 with single multiplier as an extra parameter

$$
\Delta E_{\text {tandem effect }}=3.33 * d * V_{\mathrm{T} 1 \text { foil }},
$$

where $d$ is the tabulated width (sigma) of the equilibrium charge state distribution at the specific energy (see from [16] for example), $V_{\mathrm{T} 1 \text { foil }}$ is the $\mathrm{T} 1$ foil voltage in $\mathrm{kV}$ and $\Delta E_{\text {tandem effect }}[\mathrm{keV}]$ is FWHM value of the energy spread when calculated from the ToF. The multiplier 3.33 comes from the $2.355 * \sqrt{2}$, where 2.355 is the factor to convert sigma, the value of $d$, to FWHM and $\sqrt{2}$ is the extra spreading caused when incoming ion charge state spread is quadratically summed with the charge state spread leaving from the 
$\mathrm{T} 1$ foil. The simple linear formula does not preserve at $\mathrm{T} 1$ foil $=0 \mathrm{~V}$ or similar small values for $\mathrm{T} 1$ foil voltages, but it gives reasonable values for otherwise over a wide energy, mass and T1 foil voltage ranges. In Fig. 7 it is shown for the case of $150 \mathrm{keV} \mathrm{He}$ that the T1 foil voltage has close to linear dependence down to about $1000-500 \mathrm{~V}$ voltage but then, other voltages of the timing gates contribute more to the tandem effect. In a similar manner the estimate (1) used in the Fig. 7 drops below $80 \%$ of the simulated values for $\mathrm{T} 1$ foil potentials smaller than $-500 \mathrm{~V}$.

Although small resolution degradation is experimentally seen with He energy of $250 \mathrm{keV}$, $+1000 \mathrm{~V}$ T1 toblerone voltages and maximum $\mathrm{T} 1$ foil voltage of $-1200 \mathrm{~V}$ the higher energy data points did not gave concluding evidence. This was due to the measured resolution of about $5 \mathrm{keV}$ with $\mathrm{T} 1$ foil voltage of $0 \mathrm{~V}$ for the $250 \mathrm{keV}$ He ions measured with scattered beam from a thin Au film on Si substrate. The simulated data points in Fig. $6 \mathrm{a}$ ) and b) for $\mathrm{T} 1$ foil voltage of $4 \mathrm{kV}$ give comparable results to the experimental data presented in [2]. In this reference, for $\sim 250 \mathrm{keV}$ He ions the resolution was $3 \mathrm{keV}$ when $\mathrm{T} 1$ foil was grounded and $12 \mathrm{keV}$ when $\mathrm{T} 1$ foil was $-4 \mathrm{kV}$.

From the simulations presented, especially in the Fig. 6 d), it can be concluded that the average charge state $q_{a v}$ gives no contribution to the tandem effect (for example $75 \mathrm{MeV}$ $\mathrm{Cu}$ used in Fig. $6 \mathrm{~d}$ ) has average charge state $q_{a v}$ of 18.8+). This is due to the fact that incoming ions and those leaving from the $\mathrm{T} 1$ foil have the same average charge state and no practical velocity change occurs before/after the foil. For this reason all ions accelerate before the $\mathrm{T} 1$ foil and decelerate after it (or vice versa depending on the $\mathrm{T} 1$ foil polarity) thus gaining zero average net energy in the process. The only differences to the energy and thus to the ToF comes from the width $d$ of the charge state distribution.

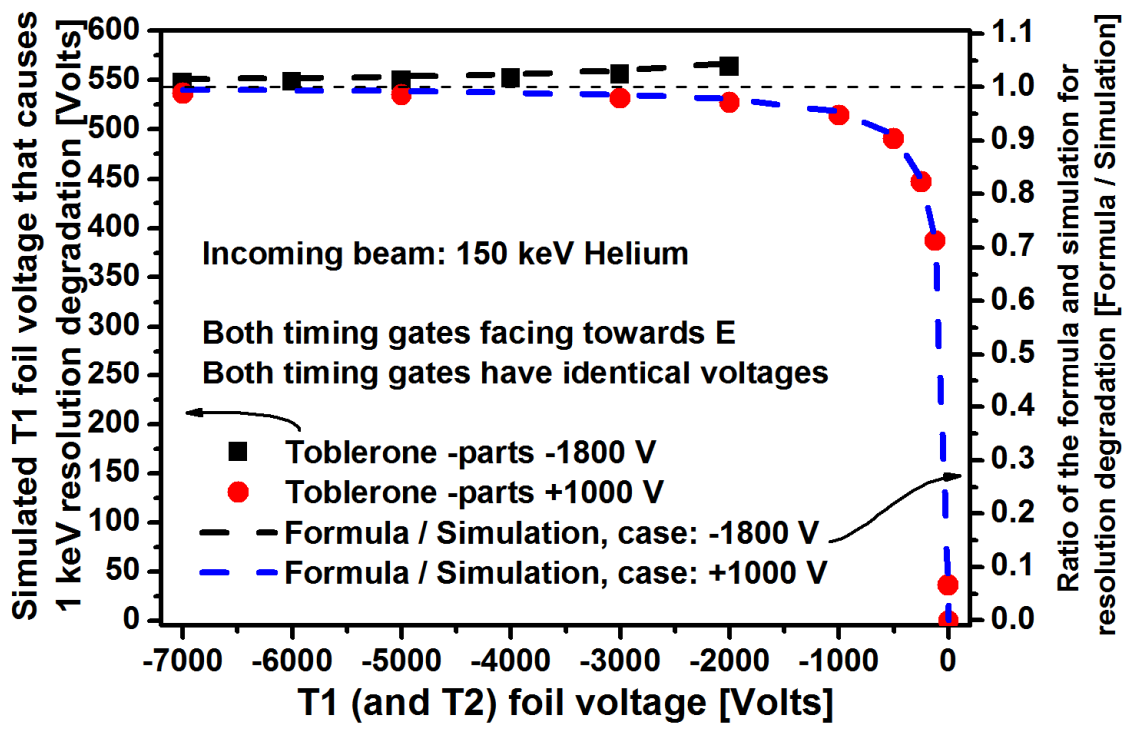

Figure 7. T1 foil voltage dependence and the accuracy of the formulation. Left scale: T1 foil voltage that causes $1 \mathrm{keV}$ degradation to the simulated energy resol ution. Right scal e: a comparison between the formulation and the simulation in the T1 foil voltage range. Right scale scaled to 1.0 where T1 foil dependence is at linear region. $150 \mathrm{keV}$ He beam 
was used with the timing gate configuration where both timing gates face towards the $E$ detector.

\section{Conclusions}

Two carbon foil time pick-up detectors were modeled using the real Jyväskylä ToF-ERD spectrometer design in the SimION program to simulate the tandem effect. With the simulations different timing gate orientations, voltages and ions and their energies were tested. The tandem effect was found to be equal for all timing gate orientations. The dominating factor, as identified also in the earlier publications was the T1 foil voltage, while other voltages had impact to the tandem effect only when the T1 foil voltage was less than $-500 \mathrm{~V}$. Simple formula to estimate the magnitude of the tandem effect was found to fit the data on wide energy, ion mass and $\mathrm{T} 1$ foil voltage range. In this estimation, the width $d$ of the equilibrium charge state probability distribution and the T1 foil voltage both have a linear dependence to the magnitude of the tandem effect. The bending of the low energy proton trajectories in the T1 mirror electric field can led to the situation where no protons reach the $\mathrm{T} 2$ foil if the solid angle of the detector is strictly

\section{Acknowledgements}

This work was supported under the auspices of Finnish Centre of Excellence Programme 2006-2014 (Project No. 213503, 251353 Nuclear and Accelerator Based Physics), 360 Finnish Funding Agency for Technology and Innovation (Tekes) project ALEBOND 361 (decision no. 40079/08), MECHALD (decision no. 40207/11) and HIUDAKE (decision 362 no. 70027/11) and Tekes EU-regional funds project (decision no. 70039/08).

\section{References}

366 [1] M.H. Mendenhall and R.A. Weller, Nucl. Instr. and Meth. B, 59 (1991), p. 120.

367 [2] M. Döbeli, R. Ender, V. Liechtenstein, D. Vetterli, Nucl. Instr. and Meth. B, 142

368 (1998), p. 417.

369 [3] Hamamatsu, MCP \& MCP assembly - selection guide,

370 http://www.sales.hamamatsu.com/resources/pdf/etd/MCPassy_TMCP0001E09.pdf

371 (25.2.213).

372 [4] Tectra, Microchannel Plates and Microchannel Plate Detectors,

373 http://www.tectra.de/MCP.htm (25.2.1013). 
374 [5] A. Zebelman, W. Meyer, K. Halbach, A. Poskanzer, R. Sextro, G. Gabor, D. Landis, 375 Nucl. Instr. and Meth., 141 (1977), p. 439.

376 [6] F. Busch, W. Pfeffer, B. Kohlmeyer, D. Schüll, F. Pühlhoffer, Nucl. Instr. and Meth., $377171(1980)$, p. 71.

378 [7] Laitinen M., Rossi. M., Julin J., T. Sajavaara, Submitted to Nucl. Instr. and Meth. B, 379 (2013).

380 [8] C.G. Drexler and R.D. DuBois, Phys. Rev. A, 53 (1996), p. 1630.

381 [9] K. Arstila, T. Sajavaara, J. Keinonen, Nucl. Instr. and Meth. B, 174 (2001), p. 163.

382 [10] S. Giangrandi, T. Sajavaara, B. Brijs, K. Arstila, A. Vantomme, W. Vandervorst, 383 Nucl. Instr. and Meth. B, 266 (2008), p. 5144.

384 [11] Z. Siketic, I.B. Radovic, M. Jaksic, N. Skukan, Rev. Sci. Instrum., 81 (2010), p. 385033305.

386 [12] Y. Zhang, B.D. Milbrath, W.J. Weber, M. Elfman, H.J. Whitlow, Appl. Phys. Lett., 38791 (2007), p. 094105.

388 [13] Simion 8.1, SIMION® Ion and Electron Optics Simulator, Scientific Instrument 389 Services, Inc.

390 [14] F. Grüner, F. Bell, W. Assmann, M. Schubert, Phys. Rev. Lett., 93 (2004), p. 391213201.

392 [15] M. Kiisk, B. Erlandsson, M. Faarinen, R. Hellborg, K. Håkansson, P. Persson, G.

393 Skog, K. Stenström, Nucl. Instr. and Meth. A, 481 (2002), p. 1.

394 [16] N. Novikov and Y. Teplova, in Journal of Physics: Conference Series (IOP

395 Publishing, 2009), p. 082032.

396 [17] J. Armstrong, J. Mullendore, W. Harris, J. Marion, P. Phys. Soc., 86 (1965), p. 1283.

397 [18] P.L. Smith and W. Whaling, Phys. Rev., 188 (1969), p. 36.

398 [19] P. Hvelplund, E. Laegsgaard, J. Olsen, E. Pedersen, Nucl. Instr. and Meth., 90 399 (1970), p. 315.

400 [20] E.J. Knystautas and M. Jomphe, Phys. Rev. A, 23 (1981), p. 679.

401 [21] K. Shima, T. Ishihara, T. Miyoshi, T. Mikumo, Phys. Rev. A, 28 (1983), p. 2162. 